\title{
SPECTRAL PROPERTIES OF A CLASS OF SINGULAR DIFFERENTIAL OPERATORS
}

\section{A. A. Kalybay, R. Oinarov and L.-E. Persson}

Abstract. We consider the operator

$$
A_{0} f=(-1)^{n} \frac{1}{v(t)}\left(D_{\rho}^{n}\right)^{*}\left[u^{2}(t) D_{\rho}^{n}\left(\frac{f(t)}{v(t)}\right)\right],
$$

where

$$
D_{\rho}^{n} f(t)=\frac{d^{k}}{d t^{k}}\left[\rho(t) \frac{d^{m} f(t)}{d t^{m}}\right],\left(D_{\rho}^{n}\right)^{*} f(t)=\frac{d^{m}}{d t^{m}}\left[\rho(t) \frac{d^{k} f(t)}{d t^{k}}\right], k+m=n .
$$

Our main aim is to prove some spectral properties of a natural extension of this operator. In order to prove this we need to prove some properties of a function space, connected to the operator $D_{\rho}^{n}$, and some embedding theorems of independent interest.

Mathematics subject classification (2000): 46E35, 47B25.

Key words and phrases: Inequalities, weights, weighted function space, weighted differential operator, spectrum, eigenvalues, spectrum discreteness, spectrum positive definiteness, resolvent nuclearity.

\section{REFERENCES}

[1] ApysheV, O.,D., AND OTElBAyEV, M., On spectrum of one class of differential operators and some embedding theorems, Izv. Acad. Sci. SSSR, Ser. Mat., 43 (1979), 739-764.

[2] Birman, M. S., On spectrum of singular boundary problems, Matem. Sbornik, 55 (97) : 2 (1961), 125-173.

[3] Glazman, I. M., Direct Methods of Qualitative Spectral Analysis of Singular Differential Operators, Moscow, Physmatgiz, 1963.

[4] Kalybay, A. A., A Generalization of the weighted Hardy inequality for one class of integral operators, Siberian Math. J. 45 (2004), iss. 1, 100-112.

[5] Kalybay, A. A., and Persson, L.-E., Three weights higher order Hardy inequalities, Function Spaces and Applications, vol. 4, 2 (2006), 163-191.

[6] Kudryavcev, L. D., On equivalent norms in weighted spaces, Trudy Mat. Inst. Steklov, 170 (1984), 161-190.

[7] Kufner, A. and Persson, L.-E., Weighted Inequalities of Hardy Type. World Scientific, New Jersey/London/Singapore/Hong Kong, 2003, 357 pages.

[8] Mynbayev, K., and Otelbayev, M., Weighted functional spaces and differential operator spectrum, Moscow, Nauka, 1988, 288 pp.

[9] Muckenhoupt, B., Hardy's inequality with weights, Stud. Math., vol. XLIV, 1 (1972), 31-38.

[10] Oinarov, R., Boundedness and compactness of superposition of fractional integration operators and their applications, Proc. Function Spaces, Differential Operators and Nonlinear Analysis (FSDONA 2004), Math. Institute, Acad. Sci., Czech Republic, (2004), 213-235.

[11] Otelbayev, M., Criteria of spectrum discreteness of one singular operator and some embedding theorems, Differential Equations, 13 (1977), N 1, 111-120. 
[12] Otelbayev, M., Criteria of resolvent kernelness of Sturm - Liouville operator, Mat. Zametki, 25 (1979), $\mathrm{N} 4,569-572$.

[13] Otelbayev, M., Estimates of specrum of Sturm - Liouville operator, Almaty, Gylym, 1990, 192 pp.

[14] Riss, F., and Sekefal'vi-Nad', B., Lectures on Functional Analysis, Moscow, Mir, 1979, 589 pp.

[15] Stepanov, V. D., Weighted inequalities of Hardy type for higher-order derivatives and their applications, Dokl. Akad. Nauk SSSR, 302 (1988), N1, 1059-1062; transl. in Soviet Math. Dokl. 38 (1989), N2, 389-393.

[16] Stepanov, V. D., Two-weighted estimates of Riemann-Liouville integrals, Izv. Akad. Nauk SSSR, Ser. Mat., 54 (1990), N3, 645-654; transl. in Math. USSR-Izv., 36 (1991), 669-681. 